\title{
Breath Figure-Assisted Fabrication of Nanostructured Coating on Silicon Surface and Evaluation of Its Antireflection Power
}

\author{
Francesco Galeotti, ${ }^{1}$ Franco Trespidi, ${ }^{2}$ and Mariacecilia Pasini ${ }^{1}$ \\ ${ }^{1}$ CNR-Istituto per lo Studio delle Macromolecole (ISMAC), Via A. Corti 12, 20133 Milano, Italy \\ ${ }^{2}$ Ricerca sul Sistema Energetico (RSE), Strada Torre della Razza, Località Le Mose, 29122 Piacenza, Italy \\ Correspondence should be addressed to Francesco Galeotti; galeotti@ismac.cnr.it
}

Received 29 December 2015; Revised 16 March 2016; Accepted 27 March 2016

Academic Editor: Shu Seki

Copyright (C) 2016 Francesco Galeotti et al. This is an open access article distributed under the Creative Commons Attribution License, which permits unrestricted use, distribution, and reproduction in any medium, provided the original work is properly cited.

We report our recent results on the fabrication of nanostructured polymer layers aimed at developing efficient antireflection coating on silicon. The proposed manufacturing approach is based on self-assembly and relies on breath figure formation. By simple and straightforward operations, we are able to produce different nanostructured coatings: densely packed nanodomes, randomly distributed nanopores, and multilayered close-packed nanopores. By optical reflectivity measurements on coated silicon wafers, we show that the latter type of nanostructure is able to reduce the reflectivity of standard silicon surface ( $\approx 40 \%$ at $450 \mathrm{~nm})$ to about $10 \%$.

\section{Introduction}

Light-matter interaction plays a crucial role in many modern technological applications, including photovoltaics and many other electrooptical devices. In order to operate efficiently, photovoltaic cells have often the need to improve the coupling of light inside a material with a high refractive index $(n)$. In the presence of a $n$ step, light transmission is limited by the naturally occurring Fresnel reflection, always present at any interface between different $n$ media. Standard semiconductor materials employed in electrooptical devices typically reflect from $30 \%$ to $50 \%$ of the incoming light at normal incidence in the visible and near-IR range. This value generally increases at higher incidence angles. Therefore, reducing the optical reflectivity in the visible and near-IR spectra by an effective antireflection (AR) coating is a key issue for increasing the efficiency of the electrooptical conversion process.

Standard multilayer coatings used in photovoltaic panels and concentrating photovoltaic systems normally reach high frontal AR power, but they are less effective in reducing the losses due to the angular dependence of the reflectivity. Nanostructures have the ability to fill this gap. In fact, realizing proper nanostructures on the semiconductor surface produces a gradual variation of the equivalent $n$ between air and the semiconductor [1-3], reducing light reflectivity.
A straightforward and simple way of producing nanostructures is provided by self-assembly, which has emerged as one of the most significant paradigms for the fabrication of new materials of technological interest because of its natural aptitude for producing complex nano- and microstructures [4-6]. The possible structures encompass a wide range of morphologies emerging from microphase separation of block copolymers [7], dewetting [8], coffee rings [9], and microsphere assembly [10]. In this scenario, breath figures (BFs) can be envisaged as a valid option [11]. In fact, by exploiting this autoorganization phenomenon, thin polymeric films having a close-packed distribution of micrometric and submicrometric cavities are readily obtainable: (i) through a single step approach, (ii) in a few seconds, and (iii) using very simple and accessible laboratory equipment. This is because BF process is a particular kind of templating method in which the template is an ordered array of water droplets that simply evaporates off at the end of the process. This is a great advantage with respect to most of the other known templating approaches, where the templates need to be removed after the fabrication of the porous films and in most cases their preparation or elimination is not trivial. For these reasons, BF is one of the most widely employed methods for the fabrication of porous polymer films [12-23]. 
We have recently demonstrated the possibility of reducing light reflection from glass surfaces by attaching on them nanopatterned PDMS thin films, obtained via BF approach $[24,25]$. In the present work, we fabricated directly on a semiconductor surface ( $\mathrm{Si}$ wafer) different nanostructured layers obtained by exploiting the same straightforward process, with the aim of reducing the reflection produced by the surface, thus increasing the amount of light transmitted into the optoelectronic device.

\section{Materials and Methods}

Poly[9,9-di(2-(2-tetrahydropyranyloxy)hexyl)fluorene-alt-9, 9-dioctylfluorene] (PFO), used for preparing the BF arrays, was synthesized in our laboratory by a properly controlled Suzuki polycondensation, according to a previously reported procedure [26]. PDMS elastomer (Sylgard ${ }^{\circledR}$ 184) was purchased from Dow Corning. As semiconductor substrate, we used a wafer of crystalline silicon.

2.1. Fabrication of the Nanostructured Coatings. For the dropcast BFs, we deposited $200 \mu \mathrm{L}$ of a $\mathrm{CS}_{2}$ solution of PFO $\left(2 \mathrm{mg} \mathrm{mL}^{-1}\right)$ on a piece of silicon wafer by using a microsyringe and let it evaporate under a flux of moist nitrogen $(80 \%$ $\mathrm{RH})$ at a flow of $150 \mathrm{~L} \mathrm{~h}^{-1}$. The process was complete in 10$20 \mathrm{~s}$ and a porous polymer film (thickness $\approx 2 \mu \mathrm{m}$ ) was left on silicon. For the spin-coated BF, a $12 \mathrm{mg} \mathrm{mL}^{-1}$ solution was dropped on the silicon substrate while spinning at $4000 \mathrm{rpm}$, and a nitrogen humid flow of $500 \mathrm{~L} \mathrm{~h}^{-1}$ at $80 \% \mathrm{RH}$ was applied on top of the spin-coating plate. For preparing the flat PFO sample, spin-coating was performed without applying the nitrogen humid flow. The final thickness of both the patterned and the flat PFO films was about $100 \mathrm{~nm}$. For the replica molding of the $\mathrm{BF}$ films, the silicon wafer coated by the nanoporous film was gently covered with $50 \mathrm{mg}$ of PDMS prepolymer/curing agent mixture, to obtain a film with average thickness of $\approx 50 \mu \mathrm{m}$, and let to rest for $1 \mathrm{~h}$ to allow the PDMS to fill the cavities. After this time, the substrate was backed in oven for $10 \mathrm{~h}$ at $60^{\circ} \mathrm{C}$. Finally, the sample was soaked in dichloromethane until the complete dissolution of the template, which releases the PDMS layer. After drying, the flexible layer was applied by the flat side to a clean piece of silicon wafer for the optical characterization. The flat PDMS sample was prepared following the same procedure, by using a nude silicon wafer as the substrate for liquid PDMS.

2.2. Optical Characterization. In this study, silicon was chosen as substrate because its $n(\approx 4)$ is sufficiently high to simulate the behaviour of typical high refractive index materials used for the upper layer of photovoltaic cells.

To evaluate the AR properties of the nanostructured layer, it would be necessary to measure the transmittance of such nanostructured coating once deposited on the substrate.

The percentage of the power transmitted inside the substrate can be written as

$$
T \%(\lambda)=100-R \%(\lambda)-S \%(\lambda)-A \%(\lambda),
$$

where $\lambda$ is the considered wavelength, $T \%(\lambda)$ is the percentage of the power transmitted into the material substrate,

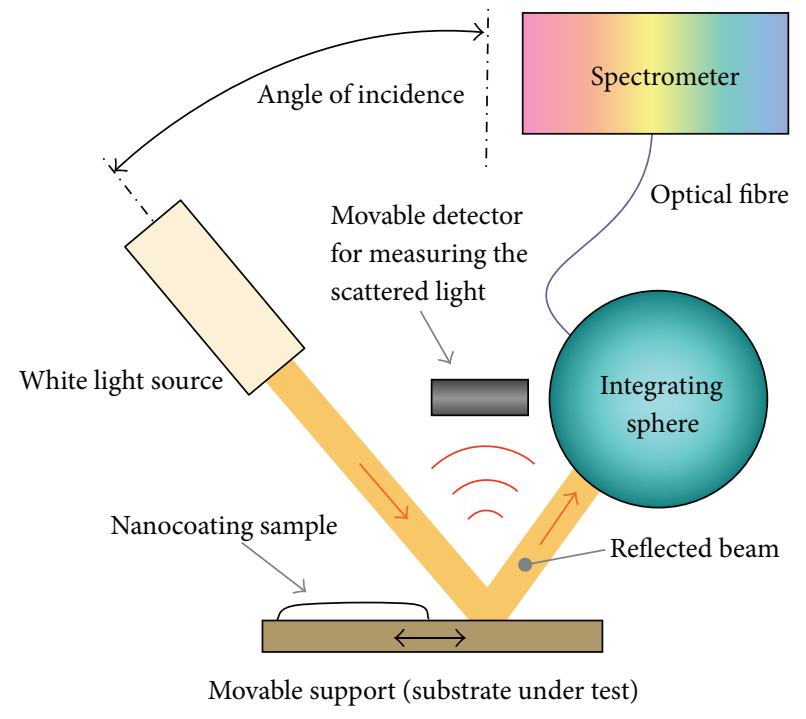

FIgURE 1: Measurement setup.

$R \%(\lambda)$ is the percentage of the power reflected from the top surface, $S \%(\lambda)$ is the percentage of the power scattered from the top surface, and $A \%(\lambda)$ is the percentage of the power absorbed by the coating layer.

Because the silicon substrate is not transparent, it is not possible to perform a direct measurement of the nanostructured coating transmission. For the thicknesses under study, the PDMS films can be considered completely transparent in the wavelength range of measurement (400-900 nm) [27], while the PFO films are transparent between 450 and $900 \mathrm{~nm}$ [28]. Therefore, in this study we generally assumed that $A \%(\lambda)=0$, by taking into account that in PFO-based coatings the initial part of the reflectivity plots $(\lambda=400-$ $450 \mathrm{~nm}$ ) is underestimated. In order to verify an increase of the transmitted power, it is sufficient to measure a reduction in the sum of the reflected and scattered powers once the coating is applied to the surface $(R \%+S \%$ in (1)). The measurement setup, shown in Figure 1, was designed to carry out the necessary measurements at different angles of incidence of the impinging radiation. A collimated light source, emitting in the visible and near-IR range, is illuminating the sample. An integrating sphere collects the reflected beam and a compact spectrometer analyses the collected light. The integrating sphere and the spectrometer are also used to monitor the incident radiation, by directing the white light beam directly to the integrating sphere. In this way, it is possible to calculate the percentage of the reflected and scattered power. The spectrum of the white light source and the spectrometer sensitivity allow measurements in the spectral range 400$900 \mathrm{~nm}$. The measurements were performed with incidence angles of the impinging beam of $30^{\circ}$ and $50^{\circ}$.

The light scattered from the sample surface is measured by an energy meter. The measurement is performed only for a certain scattering angle; however, since the surface roughness of the nanostructured coating is smaller than the impinging wavelengths, the distribution of the scattered light will follow the Rayleigh scattering theory and will have the typical shape 


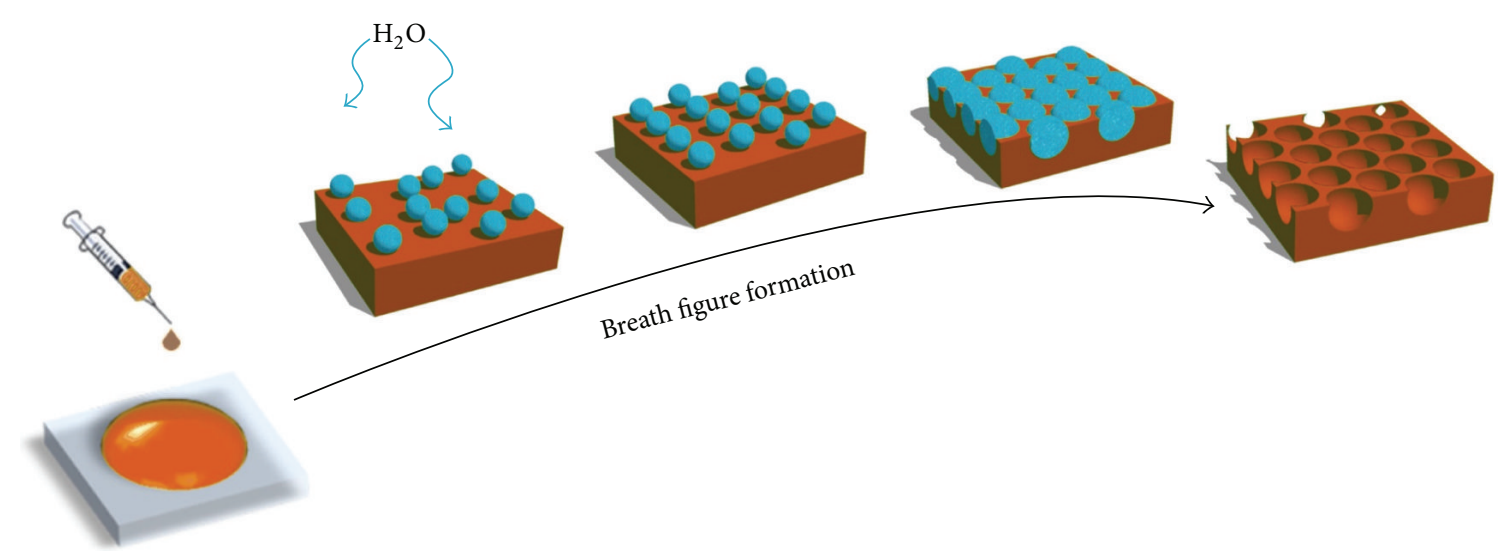

FIgURE 2: Schematic representation of breath figure formation.

of Rayleigh scattering. Therefore, it is possible to calculate the total power scattered from the surface starting from the power scattered at a certain angle. The nanostructured coating is deposited in order to cover only half area of the semiconductor. The measurements are acquired first on the nude silicon side, then the sample is moved, and a second measurement is performed on the nanostructured coating side. The two points on the semiconductor inspected are separated by few millimeters and it was verified that their optical reflectivities are equal within $0.1 \%$.

\section{Results and Discussion}

The process of BF formation relies on the precipitation of a polymer around condensed water droplets, triggered by the rapid evaporation of a polymer solution in a humid environment. Its main stages are depicted in Figure 2. As soon as the polymer solution in a volatile solvent is dropcast onto the substrate, the endothermic evaporation of the solvent lowers the system temperature, causing the water condensation. Once a droplet of water has nucleated on the polymer solution, it grows at the expense of the vapor of the surrounding atmosphere. During this stage, the growing droplets start to organize at the polymer solution/air interface into a close-packed arrangement. Once the film returns to ambient temperature, the condensed water and residual solvent evaporate, giving rise to a porous structure. When the process is adequately controlled and the key parameters (polymer concentration, kind of solvent, evaporation rate, and relative humidity) are accurately adjusted, it is possible to tune some morphology features in the final film, such as the degree of order and the distribution/size of the pores [29-31]. In fact, $\mathrm{BF}$ formation can lead to randomly distributed pores as well as to highly ordered honeycomb films, depending on the conditions used. As regards the pore size, the achievable diameter ranges from submicron to the tens of microns.

As an alternative to the standard drop-cast process, $\mathrm{BF}$ arrays can be produced by spin-coating [32-35]. By following this approach, the solvent evaporation/film forming process is much faster, and it is more likely to obtain smaller porosity, because the water droplets do not have enough time to grow. Normally, BFs produced by spin-coating are poorly ordered and can be multilayered, but the whole film thickness is reduced with respect to the drop-cast approach, because most of the material and solvent are spun off the rotating substrate.

A further possibility for making nanostructured thin films starting from the BF approach is to prepare the softlithographic replica of the nanoporous film [36, 37]. In this procedure, in fact, the BF film acts as a template for PDMS, so that the close-packed array of nanopores is replaced by an array of nanoreliefs of approximately hemispherical shape. While in the two former approaches the porous film is produced directly on the substrate surface (in our case silicon), in the latter the preformed PDMS nanostructured layer is applied on the clean silicon surface after its manufacturing has been concluded.

By following the three BF-based fabrication approaches discussed above, we prepared three different types of polymeric nanostructures. As the spin- and drop-casting material, we selected an alternated fluorene-based block-copolymer synthesized in our laboratory (PFO), which already proved to be suitable for BF arrays [25]. In this polymer, in fact, the presence of hydrophilic pendants all along the polymer chains and not only at the end of the chain, as in most amphiphilic materials, allows for the realization BF films of submicrometric porosity, hence matching the requirements for AR structures. In addition, polyfluorenes have higher $n(=1.7)$ than other standard polymers used to prepare BF films (polystyrene, $n=1.5$; PMMA, $n=1.4$ ), which makes PFO more suitable for reducing the reflectivity of $\mathrm{Si}$ surface $[38,39]$. Compared to other high refractive index polymers (conjugated materials, sulfur-containing materials, and polymer/inorganic hybrid materials) [40], PFO has the further advantages of high solubility in organic solvents and low light absorption in the visible and IR spectrum.

The fabrication routes and the resulting nanostructures are summarized by the cartoon in Figure 3. The BF technique realized by spin-coating on a piece of silicon wafer afforded a thin nanoporous film (SC-BF). By applying the BF approach by drop-cast, we obtained a similarly porous film, of higher thickness (DC-BF). Because we were interested in obtaining a submicrometric porosity suitable for AR coating purposes, we optimized the process parameter towards this target. In particular, a polymer concentration of $2 \mathrm{mg} \mathrm{mL}^{-1}$ and a moist 

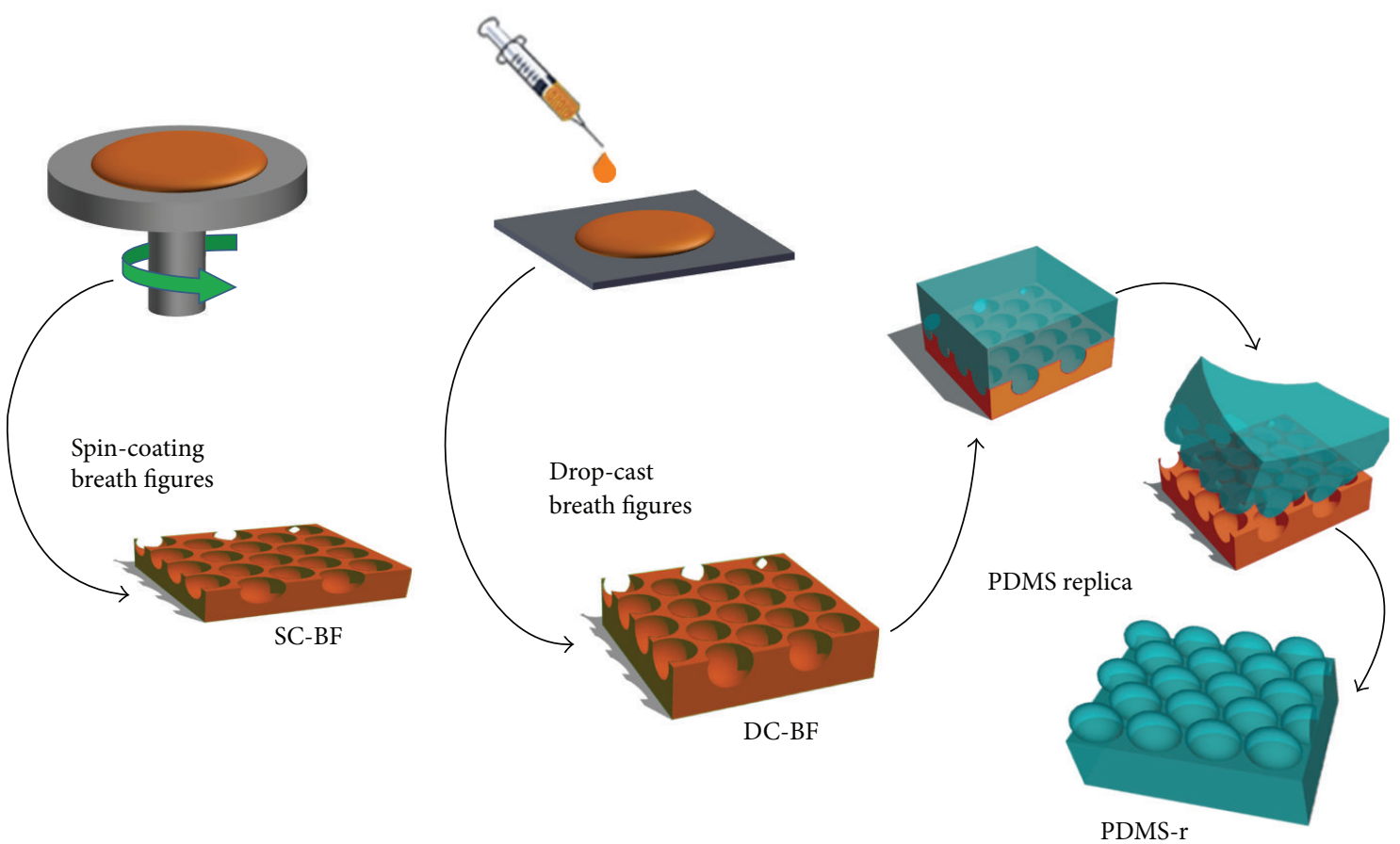

FIGURE 3: Schematic representation of the fabrication routes leading to three different types of nanostructures: SC-BF, DC-BF, and PDMS-r.

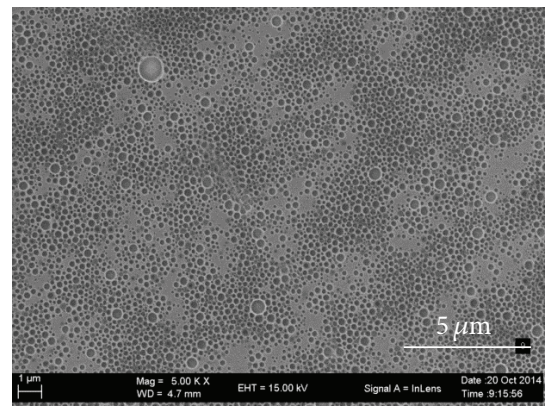

(a)

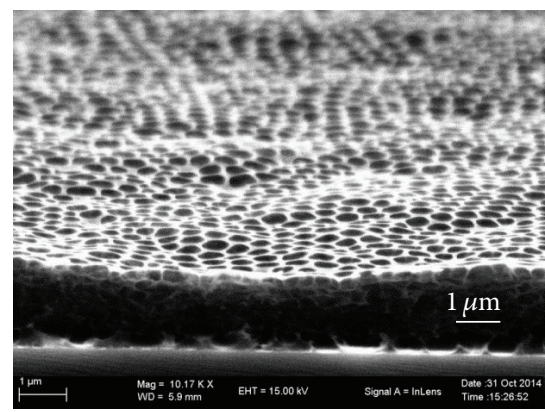

(d)

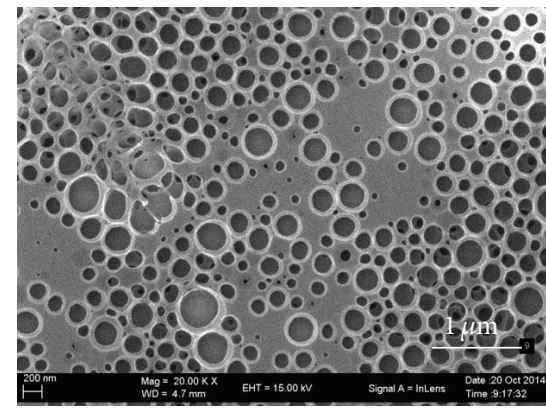

(b)

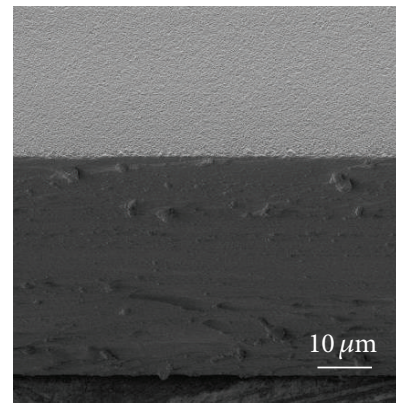

(e)

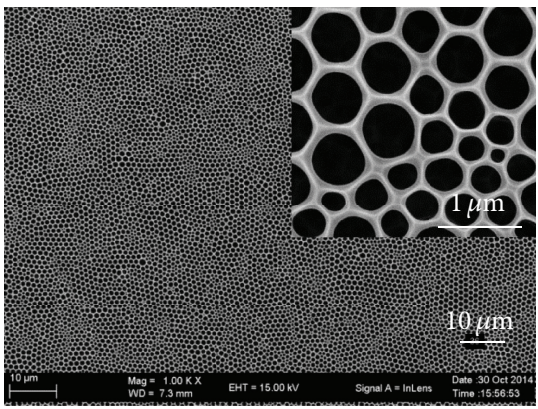

(c)

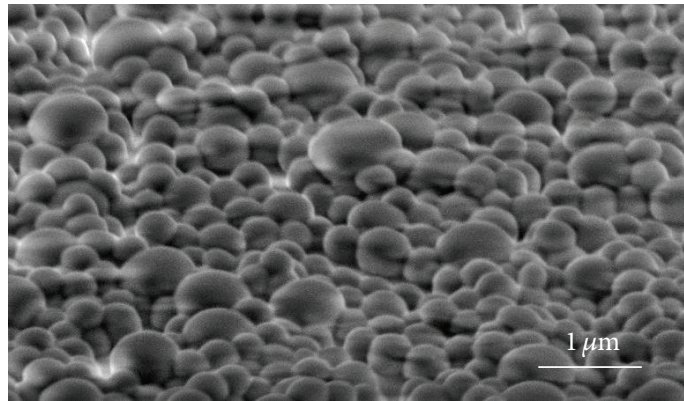

(f)

FIGURE 4: SEM views of the three selected types of nanostructures. (a)-(b) Top view of SC-BF. (c) Top view and magnified top view (inset, side $\approx 3 \mu \mathrm{M}$ ) of DC-BF and section view of the same sample (d). Section (e) and magnified tilted view (f) of PDMS-r.

nitrogen flow of $150 \mathrm{~L} \mathrm{~h}^{-1}$ were used for the drop-casting approach, whereas a higher concentration $\left(12 \mathrm{mg} \mathrm{mL}^{-1}\right)$ and flow $\left(500 \mathrm{~L} \mathrm{~h}^{-1}\right)$ were necessary to obtain the spin-coated films with proper submicrometric and close-packed porosity. Finally, by applying the soft-lithographic procedure to a BF film obtained by the previous approach, we obtained a nanopatterned PDMS replica (PDMS-r).

When observed by SEM (shown in Figure 4), the selected nanostructures revealed some clear morphological differences. The surface of sample SC-BF (frames (a)-(b)) appears 
TABLE 1: Summarized description of the nanostructured films.

\begin{tabular}{|c|c|c|c|c|c|}
\hline Sample & Method & Film thickness & Nanostructure average diameter & Fill factor & Multilayer \\
\hline SC-BF & Spin-coating breath figures & $100 \mathrm{~nm}$ & $300 \mathrm{~nm}$ & Low & No \\
\hline DC-BF & Drop-cast breath figures & $1-2 \mu \mathrm{m}$ & $450 \mathrm{~nm}$ & High & Yes \\
\hline PDMS-r & Replica molding from DC-BF & $50 \mu \mathrm{m}$ & $450 \mathrm{~nm}$ & High & No \\
\hline
\end{tabular}

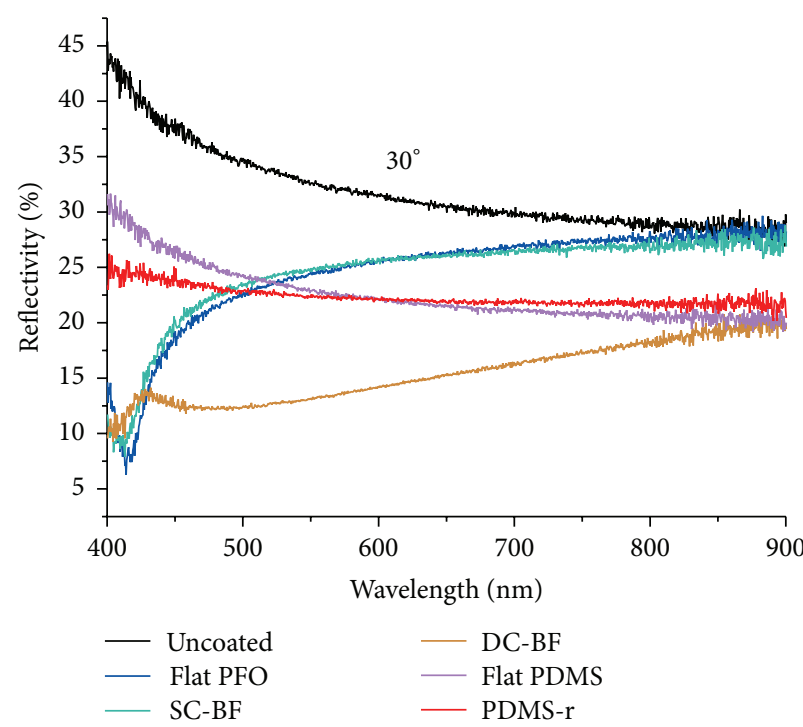

(a)

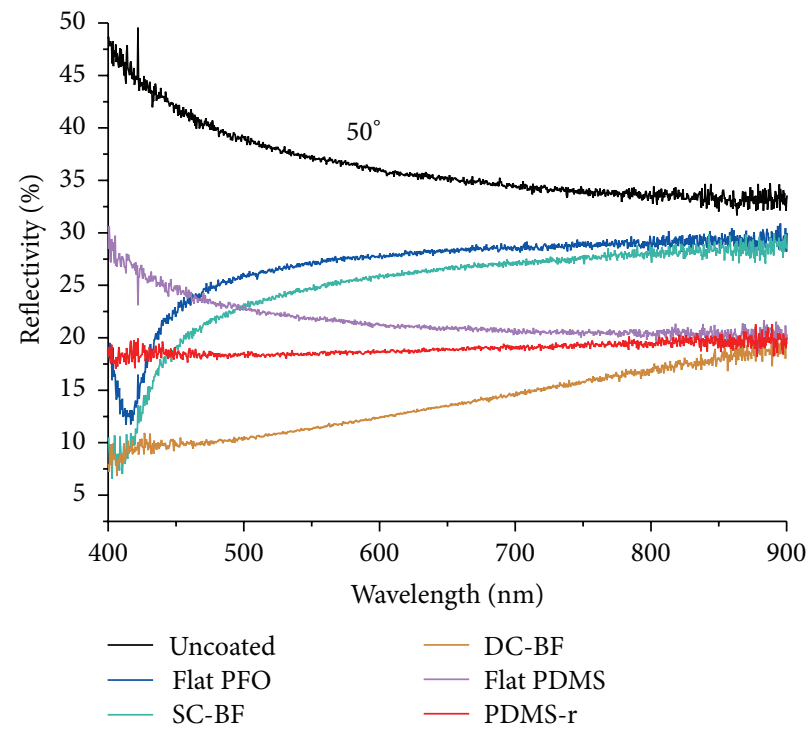

(b)

FIGURE 5: Total reflectivity of the silicon substrate (specular reflectivity + average scattered power) measured for the uncoated sample and for the different coated cases, at incidence angles of $30^{\circ}$ (a) and $50^{\circ}$ (b).

as a random distribution of close-packed pores of about $300 \mathrm{~nm}$, but the surface coverage is uncomplete, so that there are some small areas where the polymer film is flat. In the magnified SEM view (frame (b)), it is possible to distinguish the presence of some smaller pores underneath the surface pore layer. Because of the low thickness of samples produced by spin-coating (around $100 \mathrm{~nm}$ in these working conditions), we expect that mostly mono- or bilayer structures can be obtained by this approach.

In sample DC-BF, the close-packed nanopores completely cover the polymer film (frames (c)-(d)). Their size is slightly bigger than the previous case (between 400 and $500 \mathrm{~nm}$ ) and the nanostructure is clearly multilayered along the $2 \mu \mathrm{m}$ thickness of the film (see tilted view in frame (d)).

The PDMS-r sample is the positive replica of the previous nanostructure type (frames (e)-(f)). Here, hemispherical reliefs of $400-500 \mathrm{~nm}$ completely cover the surface of the PDMS layer. From the section SEM analysis (frame (e)), it is possible to notice that the height/depth of the nanostructures is much smaller than the whole thickness of the PDMS layer.

The main features of the three selected types of nanostructured films are summarized in Table 1.

To evaluate the AR performance of the fabricated nanostructures on silicon, we measured the reduction in the reflected components of light (intended as specular reflection plus scattering) produced by the presence of the coating. In fact, by assuming that absorbed component can be neglected for the coating materials in wavelength range of measurement, the amount of light transmitted into the silicon wafer can be calculated as the difference between the optical power impinging on the surface and the total reflected components, which can be measured. As discussed in the previous section, because PFO is not completely transparent for $\lambda$ values lower than $450 \mathrm{~nm}$, we must take into account that the reflectivity of PFO-based coatings is underestimated in the initial part of the spectra.

For each sample, we performed two sets of measurements by properly pointing the collimated light beam for illuminating the sample at incidence angles of $30^{\circ}$ and $50^{\circ}$. To perform a correct evaluation of the coating behaviour, it is necessary to measure also the scattered power from both the coated and the uncoated surfaces. By adding the average contribution of the scattered light to the specular reflection curves, it is possible to estimate with good approximation the real improvement produced by the different coatings.

The results of these measurements are summarized in Figure 5. For each sample, the reflectivity spectrum at the two selected angles, augmented by the average value of the measured scattered light, is presented. To better evaluate the AR effect of the nanostructures, the reflectivity of the uncoated silicon, that of the silicon coated by a flat PFO film, and that obtained by applying on silicon a flat PDMS layer have been included in the same plots. 
As expected, all the coatings reduce the reflectivity of silicon substrate. In fact, because polymers have intermediate $n$ values between those of air $(n=1)$ and silicon $(n=$ 3.8), they intrinsically work as AR layers. By comparing the nanostructured PFO obtained by spin-coating (SC-BF) with the flat PFO coating, we notice that the reflectivity reduction with respect to bare silicon is similar at $30^{\circ}$, indicating that the nanostructure has practically no effect. By contrast, the AR effect ascribable to the nanostructure is evident at $50^{\circ}$. This result suggests that the $300 \mathrm{~nm}$ porosity of sample SC-BF is partially able to provide a gradual variation of $n$ from air to silicon substrate, depending on the incidence angle.

The DC-BF nanostructure, on the other hand, shows for both incidence angles a large AR effect over the whole spectral range, with respect to both the uncoated and the flat PFO coating samples. Silicon reflectivity is decreased up to $10 \%$ by this coating, a value which is promising for practical AR applications. This indicates that the multilayered nanoporous structure evidenced in Figure $4(\mathrm{~d})$ is more effective in reducing the reflectivity of silicon than the thinner mono- or bilayer structure of sample SC-BF.

If we correlate this observation with the morphological data summarized in Table 1, we notice that the better performance shown by DC-BF sample cannot be explained by the average pore size, which is slightly bigger in this sample than in SC-BF (450 versus $300 \mathrm{~nm}$ ) and therefore should not be a favourable condition for AR. In fact, a surface pattern can work properly as graded index coating when the nanostructures are small enough with respect to the impinging wavelengths. If the nanopattern size is close to the wavelength, the resonant effects increase the scattered component of the radiation preventing a good forward transmission for all the wavelengths of the spectrum. Therefore, as regards the average pore size, the DC-BF sample should work slightly worse than the SC-BF one.

The second parameter to take into account is the fill factor of the nanostructure, which in this case results from the closepacking level of the pores. The high fill factor of DC-BF film is certainly favourable for the AR effect, because most of the light impinging the coating meets a nanostructured area, whereas the SC-BF sample, having a low fill factor, exposes a bigger fraction of flat surface to light.

The last parameter considered in Table 1 is the presence of a multilayered porous structure, as in the case of the DC-BF film. Typically, a multilayered nanoporous film can properly act as graded index coating if it has a distinct asymmetric porous structure, that is, top and bottom layer having higher and lower porosity, respectively, so that a gradual increase of density and hence of $n$ from the top to the bottom is provided [41]. Recently, Dong et al. conducted an investigation on the formation of multilayers in BF procedure applied on silicon substrates [42]. As shown by this and by other similar studies, multilayer porous films obtained by BF approach normally exhibit such asymmetric porous structure, because the cavities in the bottom of the film tend to squeeze and collapse during the BF development as the other layers are formed above [31, 42-44].

On these bases, we can speculate that the better AR performance shown by the DC-BF sample is ascribable to the combination of higher fill factor of the surface nanostructure and gradual density increase from the top to the bottom arising from asymmetrical pore size distribution of the multilayer structure. Nevertheless, at this stage of the study we cannot exclude that other optical effects, such as destructive interference or localized plasmonic resonances, could take part in the observed AR effect.

As regards the PDMS samples, our previous studies have shown that similar elastomeric nanostructured films are able to reduce light reflection on glass of nearly $50 \%[24,25]$. This result is substantially confirmed on silicon. In fact, the reflected component is reduced of about $30 \%$ and $45 \%$ at $30^{\circ}$ and $50^{\circ}$, respectively. The lower AR effect observed on silicon with respect to glass is ascribable to PDMS $n$ value (1.4) which is close to that of glass, whereas the $n$ step with silicon is much higher. In addition, the reflection plots show that the hemispherical nanostructures enhanced the AR performance of the flat PDMS layer at $50^{\circ}$, while this effect is reduced at the low incidence angle, probably because the $450 \mathrm{~nm}$ average diameter of the hemispherical reliefs is not small enough to work properly.

From the comparison of the AR behaviour of the different nanostructures discussed above, we can deduce that BF approach has the potential of being applied on devices where the reduction of light reflection is required. It is worth to remark that the first two types of presented nanostructures (SC-BF and DC-BF) are formed directly on the semiconductor surface; hence, their realization does not require a perfectly flat substrate, as for the last type (PDMS-r). Presently, in some electrooptical devices such as photovoltaic cells, the surface is not perfectly flat due to the microwires used for contacting it. BF-assisted fabrication of the AR layer could work also in those cases, without requiring planarization of the device surface.

\section{Conclusions}

In summary, we have successfully applied the breath figure approach to realize nanostructured antireflection coatings for silicon surface. By changing the fabrication conditions, we were able to select three different types of nanostructured coatings, having nanopores or nanoreliefs on their external surface. Because the proposed method is entirely based on self-assembling and soft-lithographic steps, the coating manufacturing is simple, straightforward, and does not require complicated equipment. Moreover, two of these nanostructured coating are realized directly on the semiconductor surface, which is advantageous especially in case of application to nonflat devices.

The optical characterization of silicon wafers coated by this approach revealed that the $2 \mu \mathrm{m}$ thick multilayered nanoporous film is the most effective coating of the series in reducing the reflectivity of silicon. Specifically, the amount of light reflected passes from $40 \%$ to $10 \%$ at $450 \mathrm{~nm}$, by changing from the uncoated to the coated sample, and from $22 \%$ to $10 \%$, by changing from the flat coating to the nanostructured one. Such result not only indicates that nanostructures are able to let the light enter in the semiconducting material more efficiently than a flat layer made of the same material, but 
also suggests that, after proper optimization, the proposed approach is potentially suitable for realizing AR coatings on working devices. Future work will be dedicated to transfer the fabrication approach here developed from silicon to semiconductor-based solar cells.

\section{Competing Interests}

The authors declare that they have no competing interests.

\section{Acknowledgments}

This research was supported by Accordo Quadro Regione Lombardia/CNR (decreto 3667/2013) within the project "TIMES" and by the Research Fund for the Italian Electrical System under the Contract Agreement between RSE (formerly known as ERSE) and the Ministry of Economic Development: General Directorate for Nuclear Energy, Renewable Energy, and Energy Efficiency stipulated on July 29, 2009, in compliance with the Decree of March 19, 2009.

\section{References}

[1] P. B. Clapham and M. C. Hutley, "Reduction of lens reflexion by the 'moth eye' principle," Nature, vol. 244, no. 5414, pp. 281-282, 1973.

[2] Y.-A. Dai, H.-C. Chang, K.-Y. Lai et al., "Subwavelength Si nanowire arrays for self-cleaning antireflection coatings," Journal of Materials Chemistry, vol. 20, no. 48, pp. 10924-10930, 2010 .

[3] H. K. Raut, V. A. Ganesh, A. S. Nair, and S. Ramakrishna, "Anti-reflective coatings: a critical, in-depth review," Energy \& Environmental Science, vol. 4, no. 10, pp. 3779-3804, 2011.

[4] K. Sakakibara, J. P. Hill, and K. Ariga, “Thin-film-based nanoarchitectures for soft matter: controlled assemblies into twodimensional worlds," Small, vol. 7, no. 10, pp. 1288-1308, 2011.

[5] J. H. Zhang, Y. F. Li, X. M. Zhang, and B. Yang, "Colloidal self-assembly meets nanofabrication: from two-dimensional colloidal crystals to nanostructure arrays," Advanced Materials, vol. 22, no. 38, pp. 4249-4269, 2010.

[6] G. M. Whitesides and B. Grzybowski, "Self-assembly at all scales," Science, vol. 295, no. 5564, pp. 2418-2421, 2002.

[7] D. M. Sim, M.-J. Choi, Y. H. Hur et al., "Ultra-high optical transparency of robust, graded-index, and anti-fogging silica coating derived from si-containing block copolymers," Advanced Optical Materials, vol. 1, no. 6, pp. 428-433, 2013.

[8] D. Gentili, G. Foschi, F. Valle, M. Cavallini, and F. Biscarini, "Applications of dewetting in micro and nanotechnology," Chemical Society Reviews, vol. 41, no. 12, pp. 4430-4443, 2012.

[9] H. G. Yang, M. Su, K. Y. Li et al., "Preparation of patterned ultrathin polymer films," Langmuir, vol. 30, no. 31, pp. 94369441, 2014.

[10] M. Pisco, F. Galeotti, G. Grisci, G. Quero, and A. Cusano, "Self-assembled periodic patterns on the optical fiber tip by microsphere arrays," in Proceedings of the 24th International Conference on Optical Fibre Sensors, vol. 9634 of Proceedings of SPIE, Curitiba, Brazil, September 2015.

[11] A. Muñoz-Bonilla, M. Fernández-García, and J. RodríguezHernández, "Towards hierarchically ordered functional porous polymeric surfaces prepared by the breath figures approach," Progress in Polymer Science, vol. 39, no. 3, pp. 510-554, 2014.

[12] F. Galeotti, V. Calabrese, M. Cavazzini et al., "Self-functionalizing polymer film surfaces assisted by specific polystyrene endtagging," Chemistry of Materials, vol. 22, no. 9, pp. 2764-2769, 2010.

[13] F. Galeotti, W. Mróz, G. Scavia, and C. Botta, "Microlens arrays for light extraction enhancement in organic light-emitting diodes: a facile approach," Organic Electronics, vol. 14, no. 1, pp. 212-218, 2013.

[14] M. Hernández-Guerrero and M. H. Stenzel, "Honeycomb structured polymer films via breath figures," Polymer Chemistry, vol. 3, no. 3, pp. 563-577, 2012.

[15] M. Pisco, F. Galeotti, G. Quero, A. Iadicicco, M. Giordano, and A. Cusano, "Miniaturized sensing probes based on metallic dielectric crystals self-assembled on optical fiber tips," ACS Photonics, vol. 1, pp. 917-927, 2014.

[16] F. Galeotti, A. Andicsova, S. Yunus, and C. Botta, "Precise surface patterning of silk fibroin films by breath figures," Soft Matter, vol. 8, no. 17, pp. 4815-4821, 2012.

[17] V. Vohra, A. Bolognesi, G. Calzaferri, and C. Botta, "Multilevel organization in hybrid thin films for optoelectronic applications," Langmuir, vol. 25, no. 20, pp. 12019-12023, 2009.

[18] A. Böker, Y. Lin, K. Chiapperini et al., "Hierarchical nanoparticle assemblies formed by decorating breath figures," Nature Materials, vol. 3, no. 5, pp. 302-306, 2004.

[19] L.-S. Wan, L.-W. Zhu, Y. Ou, and Z.-K. Xu, "Multiple interfaces in self-assembled breath figures," Chemical Communications, vol. 50, no. 31, pp. 4024-4039, 2014.

[20] E. Ferrari, P. Fabbri, and F. Pilati, "Solvent and substrate contributions to the formation of breath figure patterns in polystyrene films," Langmuir, vol. 27, no. 5, pp. 1874-1881, 2011.

[21] M. Du, P. Zhu, X. Yan, Y. Su, W. Song, and J. Li, "Honeycomb self-assembled peptide scaffolds by the breath figure method," Chemistry-A European Journal, vol. 17, no. 15, pp. 4238-4245, 2011.

[22] H. L. Cong, J. L. Wang, B. Yu, and J. G. Tang, "Preparation of a highly permeable ordered porous microfiltration membrane of brominated poly(phenylene oxide) on an ice substrate by the breath figure method," Soft Matter, vol. 8, no. 34, pp. 8835-8839, 2012.

[23] H. Bai, C. Du, A. Zhang, and L. Li, "Breath figure arrays: unconventional fabrications, functionalizations, and applications," Angewandte Chemie-International Edition, vol. 52, no. 47, pp. 12240-12255, 2013.

[24] F. Trespidi, G. Timò, F. Galeotti, and M. Pasini, "PDMS antireflection nano-coating for glass substrates," Microelectronic Engineering, vol. 126, pp. 13-18, 2014.

[25] F. Galeotti, F. Trespidi, G. Timò, and M. Pasini, "Broadband and crack-free antireflection coatings by self-assembled moth eye patterns," ACS Applied Materials and Interfaces, vol. 6, no. 8, pp. 5827-5834, 2014.

[26] A. Bolognesi, F. Galeotti, J. Moreau et al., "Unsoluble ordered polymeric pattern by breath figure approach," Journal of Materials Chemistry, vol. 20, no. 8, pp. 1483-1488, 2010.

[27] D. K. Cai, A. Neyer, R. Kuckuk, and H. M. Heise, "Optical absorption in transparent PDMS materials applied for multimode waveguides fabrication," Optical Materials, vol. 30, no. 7, pp. 1157-1161, 2008. 
[28] A. Perevedentsev, Y. Sonnefraud, C. R. Belton et al., "Dip-pen patterning of poly(9,9-dioctylfluorene) chain-conformationbased nano-photonic elements," Nature Communications, vol. 6, article 5977, 2015.

[29] M. H. Stenzel, C. Barner-Kowollik, and T. P. Davis, "Formation of honeycomb-structured, porous films via breath figures with different polymer architectures," Journal of Polymer Science, Part A: Polymer Chemistry, vol. 44, no. 8, pp. 2363-2375, 2006.

[30] A. J. Zhang, H. Bai, and L. Li, "Breath figure: a natureinspired preparation method for ordered porous films," Chemical Reviews, vol. 115, no. 18, pp. 9801-9868, 2015.

[31] R. Daly, J. E. Sader, and J. J. Boland, "Taming self-organization dynamics to dramatically control porous architectures," ACS Nano, vol. 10, no. 3, pp. 3087-3092, 2016.

[32] E. Servoli, G. A. Ruffo, and C. Migliaresi, "Interplay of kinetics and interfacial interactions in breath figure templating-a phenomenological interpretation," Polymer, vol. 51, no. 11, pp. 2337-2344, 2010.

[33] W. Madej, A. Budkowski, J. Raczkowska, and J. Rysz, "Breath figures in polymer and polymer blend films spin-coated in dry and humid ambience," Langmuir, vol. 24, no. 7, pp. 3517-3524, 2008.

[34] F. Pilati, M. Montecchi, P. Fabbri et al., "Design of surface properties of PET films: effect of fluorinated block copolymers," Journal of Colloid and Interface Science, vol. 315, no. 1, pp. 210222, 2007.

[35] B. W. Lim and M. C. Suh, "Simple fabrication of a threedimensional porous polymer film as a diffuser for organic light emitting diodes," Nanoscale, vol. 6, no. 23, pp. 14446-14452, 2014.

[36] Y. Xia and G. M. Whitesides, "Soft lithography," Angewandte Chemie-International Edition, vol. 37, no. 5, pp. 550-575, 1998.

[37] B. D. Gates, "Nanofabrication with molds \& stamps," Materials Today, vol. 8, no. 2, pp. 44-49, 2005.

[38] P. O. Morawska, Y. Wang, A. Ruseckas et al., "Side-chain influence on the mass density and refractive index of polyfluorenes and star-shaped oligofluorene truxenes," Journal of Physical Chemistry C, vol. 119, no. 38, pp. 22102-22107, 2015.

[39] M. Campoy-Quiles, G. Heliotis, R. Xia et al., "Ellipsometric characterization of the optical constants of polyfluorene gain media," Advanced Functional Materials, vol. 15, no. 6, pp. 925933, 2005.

[40] T. Higashihara and M. Ueda, "Recent progress in high refractive index polymers," Macromolecules, vol. 48, no. 7, pp. 1915-1929, 2015.

[41] M. Born and E. Wolf, Principles of Optics: Electromagnetic Theory of Propagation, Interference and Diffraction of Light, Cambridge University Press, 1999.

[42] R. Dong, J. Yan, H. Ma, Y. Fang, and J. Hao, "Dimensional architecture of ferrocenyl-based oligomer honeycomb-patterned films: from monolayer to multilayer," Langmuir, vol. 27, no. 14, pp. 9052-9056, 2011.

[43] M. S. Park and J. K. Kim, "Broad-band antireflection coating at near-infrared wavelengths by a breath figure," Langmuir, vol. 21, no. 24, pp. 11404-11408, 2005.

[44] L. A. Connal, P. A. Gurr, G. G. Qiao, and D. H. Solomon, "From well defined star-microgels to highly ordered honeycomb films," Journal of Materials Chemistry, vol. 15, no. 12, pp. 1286-1292, 2005. 

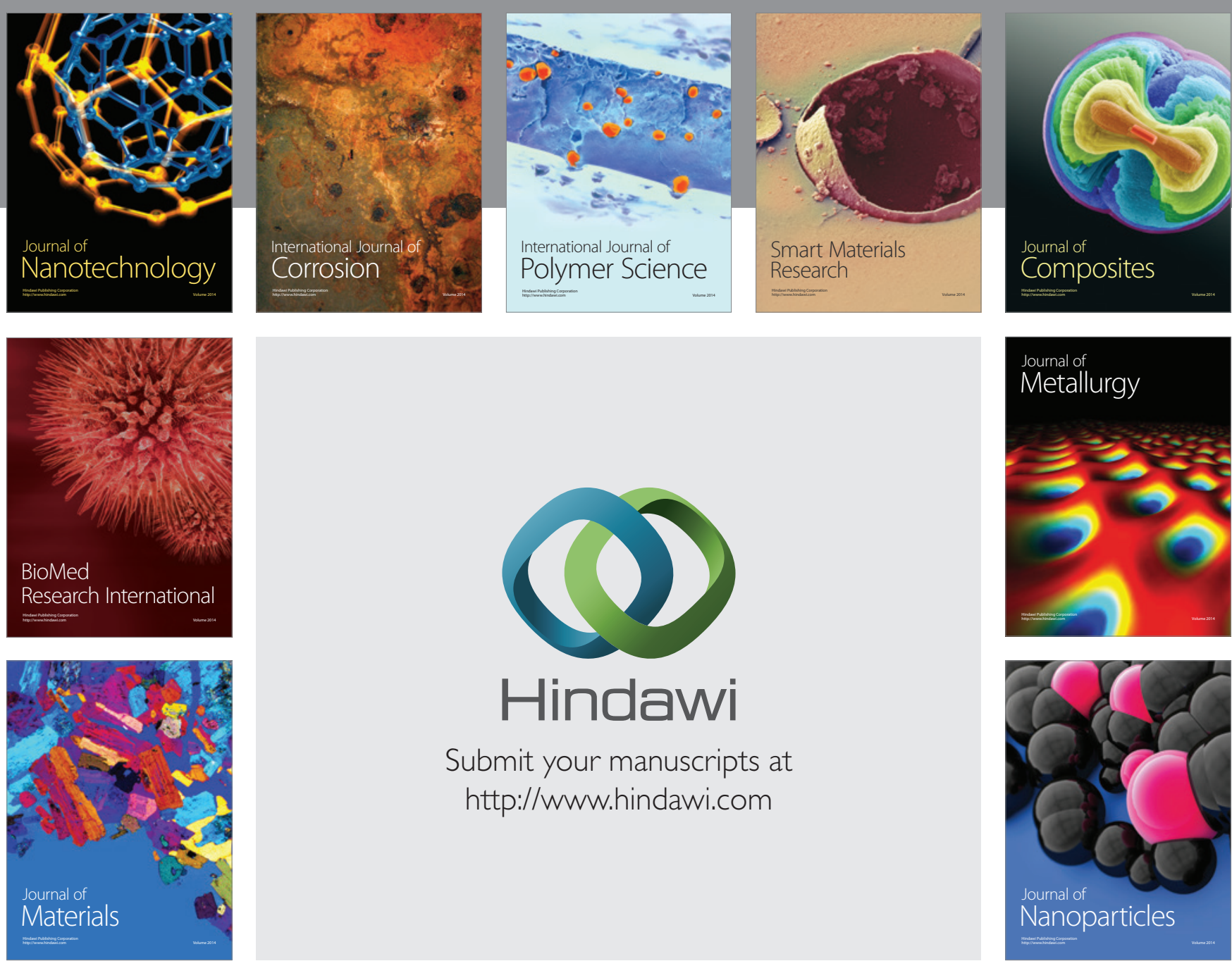

\section{Hindawi}

Submit your manuscripts at

http://www.hindawi.com

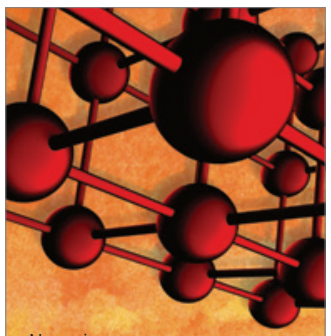

Materials Science and Engineering
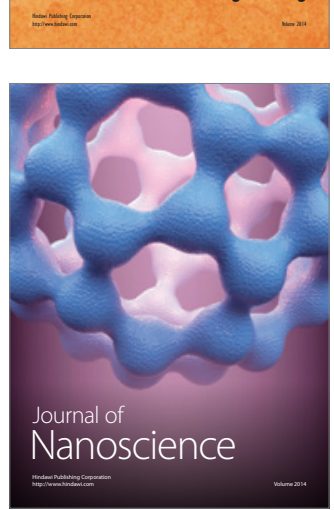
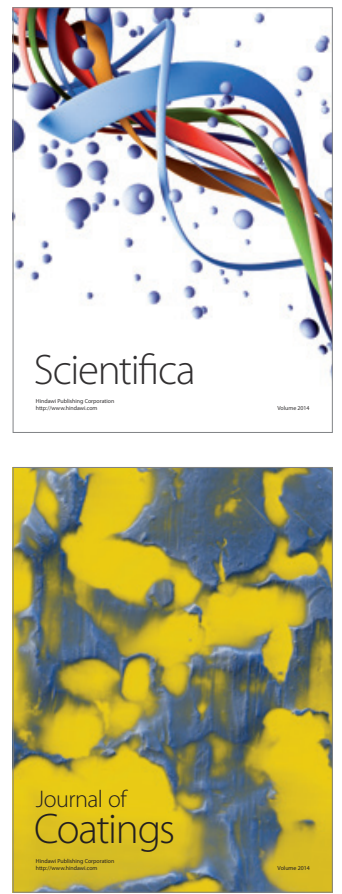
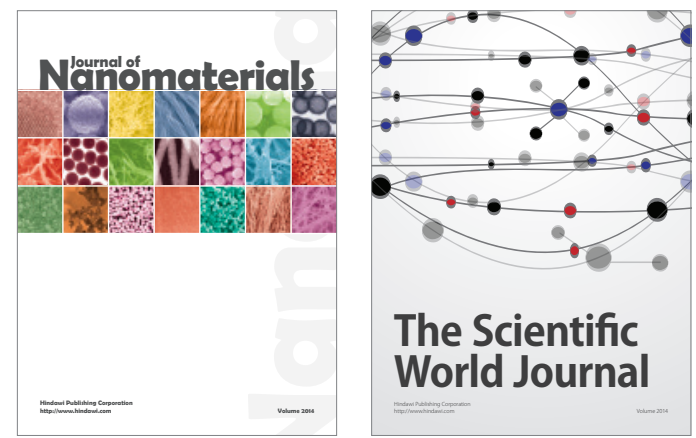

The Scientific World Journal
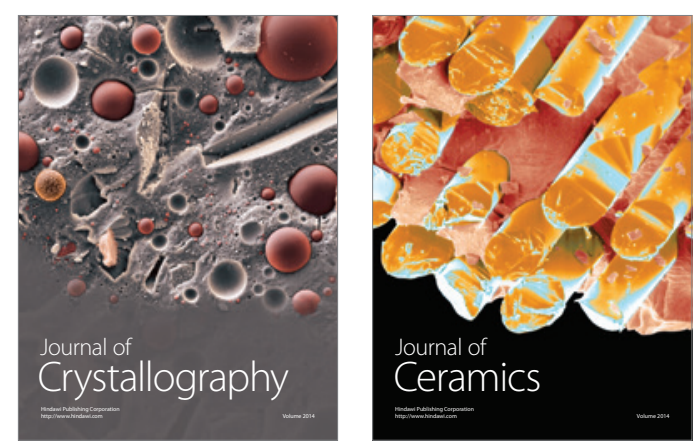
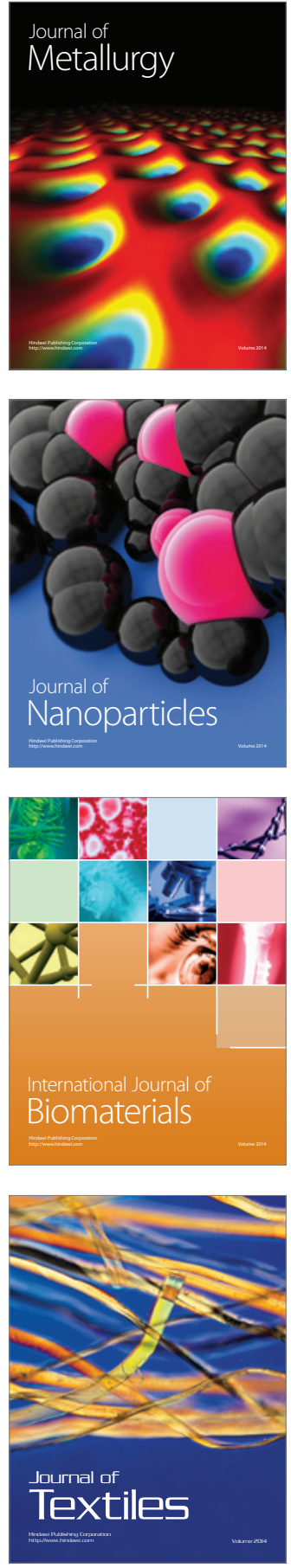